\title{
Functional and structural characterization of interactions between opposite subunits in HCN pacemaker channels
}

Mahesh Kondapuram $^{1 \#}$, Benedikt Frieg ${ }^{2 \#}$, Sezin Yüksel ${ }^{1}$, Tina Schwabe ${ }^{1}$, Christian Sattler ${ }^{1}$, Marco Lelle ${ }^{1}$, Andrea Schweinitz ${ }^{1}$, Ralf Schmauder ${ }^{1}$, Klaus Benndorf ${ }^{1}$, Holger Gohlke ${ }^{2,3^{*}}$, and Jana $\mathrm{Kusch}^{1 *}$

${ }^{1}$ Universitätsklinikum Jena, Institut für Physiologie II, Jena, Germany

${ }^{2}$ John von Neumann Institute for Computing (NIC), Jülich Supercomputing Centre (JSC), and Institute of Biological Information Processing (IBI-7: Structural Biochemistry),

Forschungszentrum Jülich GmbH, Jülich, Germany.

${ }^{3}$ Institut für Pharmazeutische und Medizinische Chemie, Heinrich-Heine Universität Düsseldorf, Düsseldorf, Germany.

\section{WORKING TITLE: Interaction of opposite subunits in HCN channels}

KEYWORDS: HCN channel, cAMP, subunit interaction, patch-clamp technique, confocal patch-clamp fluorometry, molecular dynamics simulations

${ }^{\#}$ Both authors contributed equally.

*Correspondence and material requests should be addressed to: (J.K.) Kollegiengasse 9, 07743 Jena, Germany, Phone (+49) 3641 9397668, E-mail: jana.kusch@med.uni-jena.de; (H.G.) Universitätsstr. 1, 40225 Düsseldorf, Germany, Phone: (+49) 21181 13662, Fax: (+49) 21181 13847, E-mail: gohlke@uni-duesseldorf.de

\section{Author ORCID}

Benedikt Frieg: 0000-0002-7877-0262

Klaus Benndorf: 0000-0002-0707-4083

Holger Gohlke: 0000-0001-8613-1447

Jana Kusch: 0000-0001-7206-8133 


\begin{abstract}
Hyperpolarization-activated and cyclic nucleotide $(\mathrm{HCN})$ modulated channels are tetrameric cation channels. In each of the four subunits, the intracellular cyclic nucleotide-binding domain (CNBD) is coupled to the transmembrane domain via a helical structure, the C-linker. Highresolution channel structures suggest that the $\mathrm{C}$-linker enables functionally relevant interactions with the opposite subunit, which might be critical for coupling the conformational changes in the CNBD to the channel pore. We combined mutagenesis, patch-clamp technique, confocal patch-clamp fluorometry, and molecular dynamics simulations to show that residue K464 of the C-linker is essential for stabilizing the closed state of the mHCN2 channel by forming interactions with the opposite subunit. MD simulations revealed that both cAMP and K464E induce a rotation of the intracellular domain relative to the channel pore, weakening the autoinhibitory effect of the unoccupied CL-CNBD region. The adopted poses are in excellent agreement with structural results.
\end{abstract}




\section{INTRODUCTION}

HCN (Hyperpolarization-activated and cyclic nucleotide-modulated) channels are nonselective cation channels that mediate critical neuronal and cardiac processes, including the generation of electrical rhythmicity, synaptic plasticity, somatic sensibility (reviewed in (1)), and shaping of cardiac action potentials (2). Structurally, they belong to the superfamily of voltage-gated potassium channels. In contrast to most members of this family, however, HCN channels are gated by a dual mechanism, combining two stimuli: hyperpolarization and cyclic nucleotide-binding (3-5). Hyperpolarizing voltage alone can activate the channel, whereas binding of cyclic nucleotides, such as cAMP or cGMP, has only a modulatory effect on activation: It shifts the steady-state activation relationship to more depolarized voltages, increases the maximum current amplitude, accelerates the activation kinetics, and decelerates the deactivation kinetics.

HCN channels are tetramers. Each of the four subunits consists of a membrane portion, formed by six transmembrane domains ( $\mathrm{S} 1$ to S6), with a pore region between S5 and S6, and S4 as the central part of the voltage sensor. The intracellular N-terminus harbors an $\alpha$-helical structure prior to S1, the HCN domain (6). The intracellular C-terminus carries the cyclic nucleotidebinding domain (CNBD), which is connected to the S6 helix via another $\alpha$-helical structure, the C-linker (CL) (7). Several studies suggested an autoinhibitory effect of the unoccupied CNBDCL portion, hindering a full activation of the channel. Binding of cyclic nucleotides to the CNBD releases autoinhibition and maximizes activation (8-10).

Different techniques including electrophysiological approaches, fluorescence microscopy and cryo-electron microscopy combined with mutagenesis were used to show intensive interactions between neighboring subunits: S4-S5 loop-CL interactions $(6,11)$, CL-CL interactions (12-14), CNBD-CNBD interactions $(6,12)$, and interactions between the newly discovered HCN domain with the CNBD and the voltage sensing domain $(6,15)$.

In a recent study, employing mutated murine HCN2 (mHCN2) concatamers with a defined number and stoichiometry of functional and disabled binding sites, we showed that cAMP occupation of two subunits in trans position led to a deceleration of deactivation to a similar extent as in a fully occupied wild type channel (16). By contrast, cAMP occupation of two subunits in cis position did not show any decelerating effect (16). These data raised the question of whether a direct interaction between opposite subunits is essential for channel gating. This suggestion is supported by latest high-resolution structures of HCN channels suggesting that the C-linker enables functionally relevant interactions with the opposite subunit, which might 
also be critical for coupling the conformational changes in the CNBD to the channel pore (6, $15,17)$.

Here, we address this question by combining mutagenesis, electrophysiology, confocal patchclamp fluorometry (cPCF), and molecular dynamics (MD) simulations for studying the mHCN2 channel. We show that the positively charged amino acid K464 at the hinge between A'- and B'-helix of the CL (also known as elbow structure (14)) is essential for stabilizing the closed state of the channel, most likely by direct interactions with the opposite subunit. Regarding the widely accepted gating mechanism, which proposes a rotation of the CL-CNBD relative to the channel pore to unwrap the S6 helix bundle at the intracellular side of the membrane $(18,19)$, we suggest that the interactions involving K464 hinder such a rotation. Conclusively, breaking this bond by substituting K464 promotes a rotation of the CL-CNBD relative to the channel pore, thereby destabilizing the closed conformation. 


\section{RESULTS}

\section{D HCN channel structure suggests proximity between opposite subunits}

Previous studies on subunit interactions during HCN channel gating focused on inter-subunit interactions between two adjacent subunits (e.g. $(12,20)$. Visual inspection of the recently published 3D structure of the human HCN1 (hHCN1) channel (6) revealed potential interactions also between opposite subunits. Since to date no 3D structure of a full-length HCN2 channel is available, we built homology models of the mHCN2 channel in apo and cAMPbound form based on the 3D structure of the homologous hHCN1 (Figure 1A; sequence identity $80 \%$; the mHCN2 model was built for the sequence from L136 to D650) to predict interactions between nearby residues of opposite subunits that may be essential for channel gating.

We identified a hydrogen bond between the side-chain of K464, located in the elbow of the CL of subunit $i$, and the backbone carbonyl oxygen of M155, found in the second $\alpha$-helix of the $\mathrm{HCN}$ domain $(\mathrm{HCNb})$ of subunit $i+2$ (Figure 1A,B). K464 is highly conserved among the four mammalian $\mathrm{HCN}$ isoforms (HCN1 to $\mathrm{HCN} 4)$ and spHCN from sea urchin sperm and was also found in some mammalian and invertebrate cyclic nucleotide-gated channels (Figure 1C). Channels without a positive charge at that very position carry a positive charge (lysine or arginine) one or two positions adjacent to that (Figure 1C). M155 is conserved in HCN1, $\mathrm{HCN} 2$, and HCN4. HCN3 and spHCN carry the hydrophobic amino acids valine and leucine in that position, respectively (Figure 1D). Together, from the structural and sequence analyses, the question arises whether the interactions between opposite subunits are crucial for the functional integrity of $\mathrm{HCN}$ channels. 
A

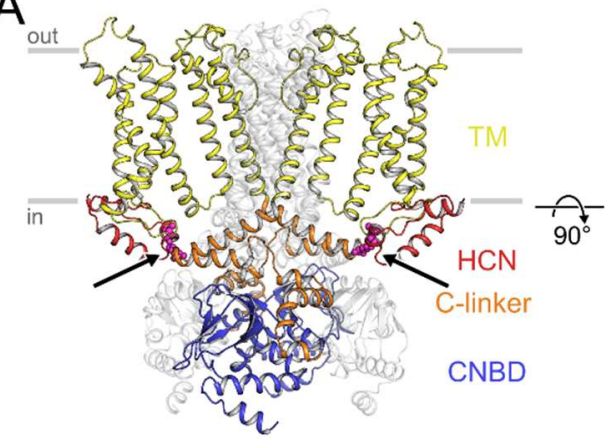

C

$\begin{array}{rr} & \\ \text { hHCN1 } & 388 \\ \text { mHCN1 } & 377 \\ \text { mHCN2 } & 430 \\ \text { mHCN3 } & 340 \\ \text { mHCN4 } & 508 \\ \text { mCNGA1 } & 383 \\ \text { spHCN (sea urchin) } & 458 \\ \text { mCNGA2 } & 368 \\ \text { mCNGA3 } & 335 \\ \text { TAX-2 (C. elegans) } & 450 \\ \text { TAX-4 (C. elegans) } & 405 \\ \text { dmCNGA } & 341 \\ \text { dmCNGl } & 493 \\ \text { SthK } & 213 \\ \text { Llik } & 226 \\ & \\ & \\ \text { hHCN1 } & 93 \\ \text { mHCN1 } & 82 \\ \text { mHCN2 } & 135 \\ \text { mHCN3 } & 46 \\ \text { mHCN4 } & 213 \\ \text { DHCN (sea urchin) } & 166\end{array}$

S6 domain

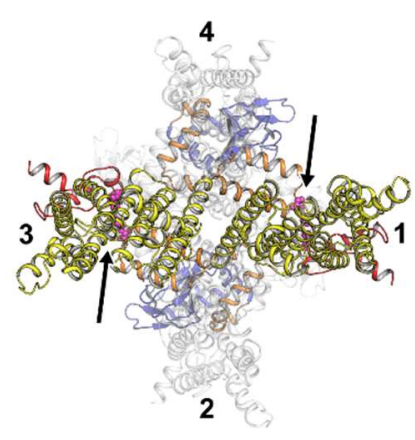

B

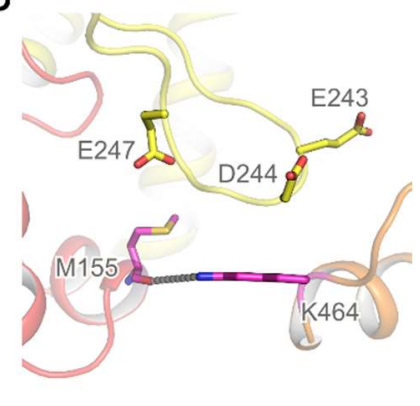

A' helix

elbow

$B^{\prime}$ helix

FVGHATAL IQSLDSSRRQYQEKYKQVEQYMSFHKLPADMRQK I HDYYEHRYQ 440 MF VGHATAL I QSLDS SRRQYQEKYKQVEQYMSF HKL PADMRQK I HDYYEHRYQ 429 430 MF I GHATAL I QSLDSSRRQYQEKYKQVEQYMSF HKL PADFRQK I HDYYEHRYQ 482 340 MF I GHATAL I QSLDSSRRQYQEKYKQVEQYMSFHKL PADTRQR I HEYYEHRYQ 392 MF I GHATAL I QSLDS SRRQYQEKYKQVEQYMSFHKLPPDTRQR I HDYYEHRYQ 560 LF I GHATNL I QSMDSSSRQYREKL KQVEEYMQYRKL PSHLRNK I L DYYEYRYR 510

83 T I VGN I GSM I SNMNAARAEFQSRVDA I KQYMNFRNVSKDMEKRV I KWF DYLWT 435 T I VGNVGSM I SNMNATRAEFQAK I DAVKHYMQFRKVSKDMEAKV I KWF DYLWT 420 335 T IVGNVGSMI SNMNAPRVEFQAK I DSVKQYMQFRKVTKDLETRV I RWFDYLWA 387 50 LLLGQ I RD I VSNANRNREEFQRKMDL ALGECKKL GL KMETTNRVRDWF I YTWQ 502 405 T I VGNVGSM I SNMSAARTEFQNKMDG I KQYMEL RKVSKQLE I RV I KWF DYLWT 457 I I VGN I GSM I SNMNVARVEFQNRMDGVKQYMAFRRVGHELEARV I RWFAYTWS 393 493 T I VGQVGNV I TNRNANRLEFERL LDGAKTYMRHHKVPGGMKRRVLRWYDYSWS 545 3 LVIGN IASL VSKLDAAKLLHRERVERVTAFLSYKRISPELQRRI IEYFDYLWE 265 6 TVIGNIAS ILGSLDLAKAAQRKKMAQVDSFLKARN I SQN I RRRVRDYYMY I ID 278

Figure 1: Identifying amino acids in potential opposite subunit interactions.

A: Structural model of the homotetrameric mHCN2 channel (residues L136 to D650). The left panel shows a view from the side, and the right panel shows a view from the top. The gray bars depict the approximate location of the membrane bilayer. Two opposite subunits are either colored according to the domain organization with the HCN domain colored red, the transmembrane (TM) domain yellow, the CL domain orange, and the cyclic nucleotide-binding domain (CNBD) blue, or in full white. M155 and K464, residues suggested to form interactions between two opposite subunits, are depicted as magenta spheres and highlighted by an arrow. B: A close-up view shows an overlay of the mHCN2 model. M155, E243, D244, E247, and K464 are depicted as sticks. The interaction between K464 and M155 is depicted as a gray dotted line. C: Sequence alignment for CNBD channels. Positions equivalent to K464 in mHCN2 are highlighted in yellow. D: Sequence alignment for $\mathrm{HCN}$ channels carrying an $\mathrm{HCN}$ domain. Positions equivalent to M155 in mHCN2 are highlighted in yellow. 


\section{K464 is involved in autoinhibition and cAMP-triggered gating enhancement}

To study the function of the highly conserved K464, we constructed two mHCN2 channel mutants, K464E for charge reversal and K464A for charge neutralization. We performed patchclamp experiments in the inside-out configuration using excised macropatches from Xenopus laevis oocytes and compared the gating of these two mutants with that of mHCN2 wild type channels. Both mutants led to robust currents. The results are summarized in Figure 2.

Channel activation was studied by applying voltage families from -70 to $-150 \mathrm{mV}$ in $10 \mathrm{mV}$ increments as shown exemplarily for HCN2 channels in the left (cAMP-free) and middle panel (saturating [cAMP] of $10 \mu \mathrm{M}$ ) in Figure 2A. Steady-state activation relationships were obtained from tail currents at a test pulse of $-100 \mathrm{mV}$, following the varying activating pulses (Figure 2B). The Boltzmann equation (eq. (1)) was fitted to relative tail current amplitudes of individual recordings, yielding the voltage of half-maximal activation, $V_{1 / 2}$, and the effective gating charge, $z \delta$. As expected from the literature, in HCN2 wild type channels the application of cAMP led to a pronounced shift of the steady-state activation relationship to more depolarized voltages. By contrast, in K464E, the relationships in both the absence and presence of saturating [cAMP] largely superimposed with the relationship obtained from wild type channels in the presence of saturating [cAMP]. Consequently, in K464E, the effect of cAMP was significantly reduced (Figure 2B) with $\Delta V_{1 / 2}=5.24 \pm 1.1 \mathrm{mV}$ in comparison to mHCN2, which showed $\Delta V_{1 / 2}=17.9 \pm 1.1 \mathrm{mV}$ (Figure 2H).

To evaluate the activation kinetics, we fitted an exponential function (eq. (2)) to the time courses of activating currents, yielding the time constant of activation, $\tau_{\text {act. }}$ The results are summarized in Figure 2C. Analogous to the results under steady-state conditions, in K464E the time constants of activation in both the presence and absence of saturating [cAMP] were largely similar to the time constant obtained from wild type mHCN2 in the presence of saturating [cAMP]. The effect of cAMP, particularly at less negative voltages, was reduced in comparison to HCN2 wild type channels, too. For studying the deactivation, we used a protocol shown in the two right panels of Figure $\mathbf{2 A}$, with varied activating hyperpolarizing voltages and a deactivating voltage step to $-30 \mathrm{mV}$. An exponential function (eq. (2)) was fitted to the deactivation time course, yielding the time constant of deactivation, $\tau_{\text {deact }}$ (Figure 2D). Again, analogous to the steady-state data and the activation kinetics, the time constants of deactivation in both the presence and absence of saturating [cAMP] were similar to the time constant obtained from $\mathrm{mHCN} 2$ in the presence of saturating [cAMP].

The effective gating charge, $z \delta$, was significantly changed by mutating K464 (Figure 2I), indicating an effect not only on cAMP-dependent but also on voltage-dependent gating. 
The same analysis was repeated for K464A (Figure 2E-I). For this construct, both steady-state activation relationships, in the absence and in the presence of cAMP, were shifted only slightly to more depolarized voltages (Figure 2E) with an only slightly reduced $\Delta V_{1 / 2}(15.0 \pm 1.6 \mathrm{mV})$ (Figure 2H). Closer inspection of the activation and deactivation kinetics revealed that this slight stabilization of the open state is not caused by promoted activation (Figure 2F) but by decelerated deactivation (Figure 2G). The effective gating charge, $z \delta$, was changed to a similar extent as for K464E (Figure 2I).

In summary, the data show that K464 stabilizes the closed (autoinhibited) mHCN2 conformation, thereby affecting both the activation and the deactivation pathway. The similar data obtained for wild type at saturating [cAMP] and K464E without cAMP led us to speculate that apo K464E channels adopt a similar conformation as cAMP-bound wild type channels. 
A

cAMP free

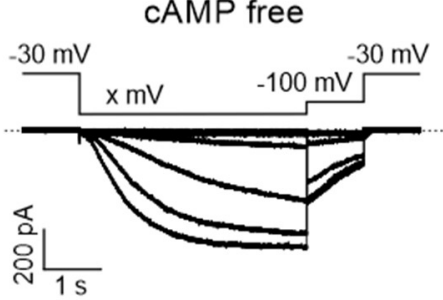

B

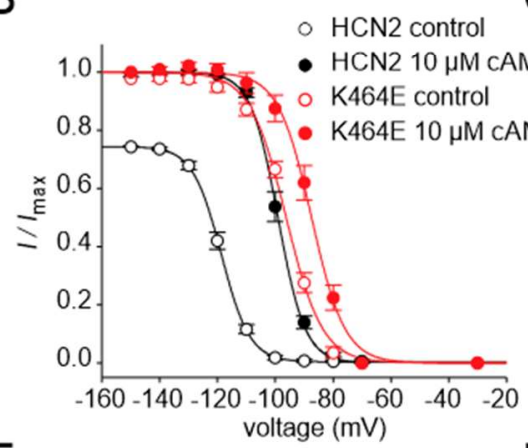

$E$

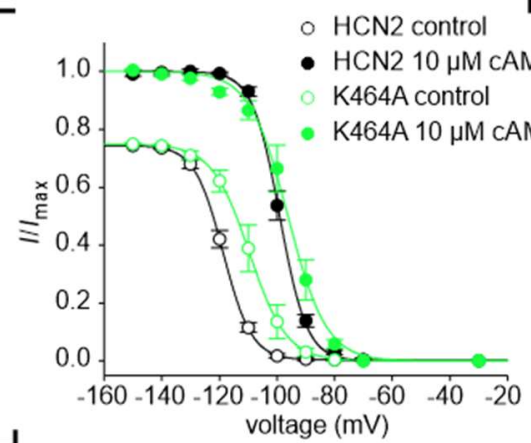

$\mathrm{H}$

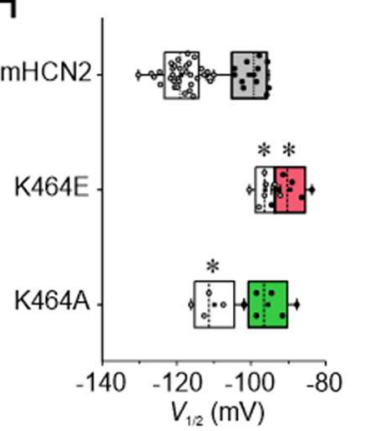

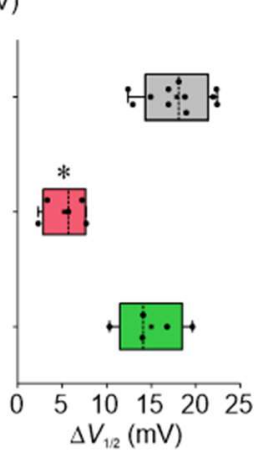

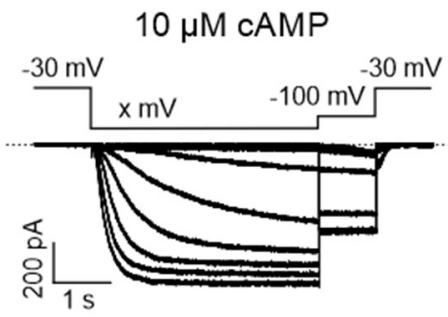

$\mathrm{C}$

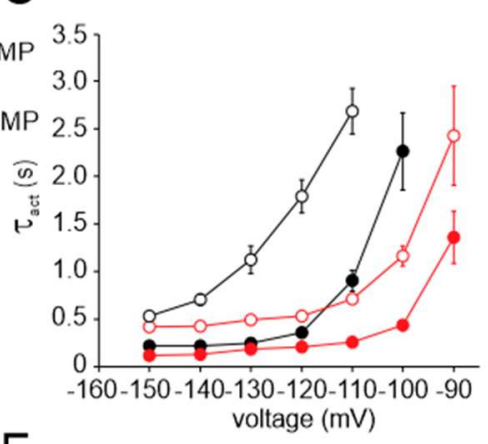

$\mathrm{F}$

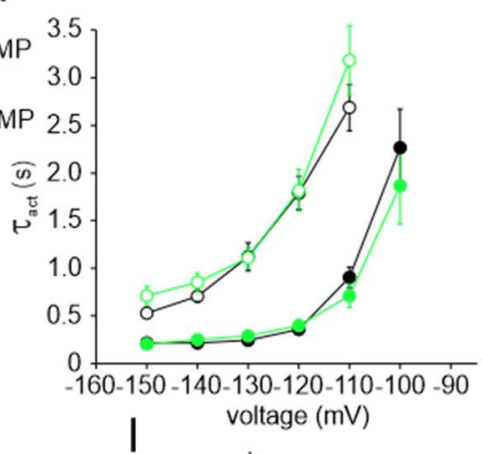

$D$
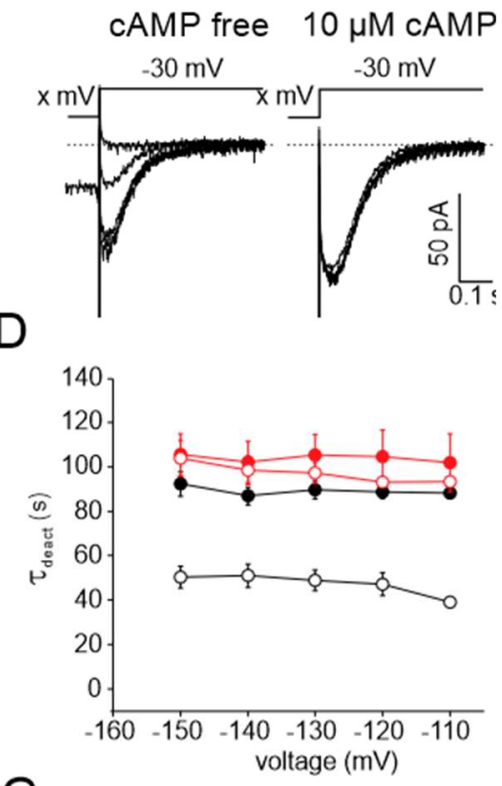

G

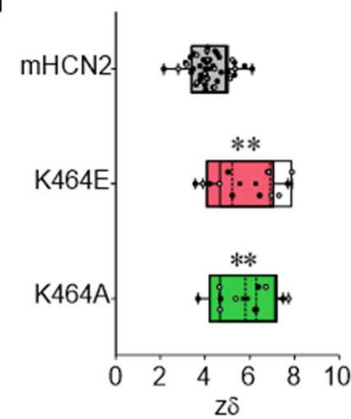

Figure 2: Voltage-dependent activation of K464 mutants at zero and saturating [cAMP] (10 $\mu$ M).

A: Exemplary current traces for mHCN2 activation. Left panel: protocol and representative traces for measuring steady-state activation and activation kinetics at zero [cAMP], middle panel: as left panel but at saturating [cAMP] $(10 \mu \mathrm{M})$, right panel: protocol and traces for measuring deactivation kinetics. B, E: Steady-state activation for K464E and K464A at zero and saturating [cAMP] in comparison to mHCN2, respectively. Solid lines indicate the result of a Boltzmann fit (equation 1) $(n=5$ to 9). C, F: Activation kinetics at zero and saturating [cAMP] for K464E, K464A, and $\mathrm{mHCN} 2(n=5$ to 9$)$. D, G: Deactivation kinetics at zero and saturating [cAMP] for K464E, K464A, and mHCN2 ( $n=5$ to 7). H: Mean $V_{1 / 2}$ values and cAMP-induced shift of $\Delta V_{1 / 2}$ for all constructs ( $\mathrm{n}=5$ to 33$)$. I: Effective gating charge $z \delta$ for all constructs. In all panels open symbols represent cAMP-free, filled symbols saturating cAMP conditions ( $\mathrm{n}=5$ to 33$)$. $\mathbf{H}$ and $\mathbf{I}$ : Asterixes indicate significant difference between the respective mutant data and mHCHN2 data under the same cAMP condition. In all box plots dotted center lines represent median, box limits represent standard deviation, whiskers represent minimum and maximum values, cirles represent individual recordings, squares represent mean. 


\section{K464 stabilizes the closed state by connecting two opposite subunits}

To corroborate this hypothesis, we performed 20 independent replicas of unbiased MD simulations of $1 \mu$ s length each of apo wild type mHCN2, wild type mHCN2 bound to four cAMP molecules, and the apo K464E variant. All simulations were analyzed towards hydrogen bond and salt-bridge interactions involving the residue K464, as shown in Figure 3B.

As in wild type mHCN2, K464 forms a hydrogen bond with the backbone of M155 in the HCN domain (in $23.7 \% \pm 2.5 \%$ of all conformations), thereby bridging two opposite subunits. This hydrogen bond frequency is only marginally reduced upon cAMP binding (n.s. rel. to apo wild type) (Figure 3A). During the MD simulations of K464E, by contrast, no hydrogen bonding with the backbone of M155 was recorded: The minimal distance between any of the side-chain oxygens in K464E to any backbone atom of M155 is usually > $5 \AA$ (Figure S1). Thus, the direct interaction between two opposite subunits mediated by K464 is most likely completely lost in the case of the K464E channel.

In K464E without cAMP, a leftward rotation (when viewed from the channel pore) of the CLCNBD relative to the channel pore is observed, as induced by cAMP binding to homologous $\mathrm{HCN}$ channels (6) (Figure 3B, C). The rotation angle in $\mathrm{K} 464 \mathrm{E}$ is $2.9^{\circ}$ on average relative to the apo wild type channel, slightly larger than that observed in MD simulations of cAMP-bound wild type mHCN2 and intermediate to rotation angles found in experimental HCN1 and HCN4 structures. As the hydrogen bond between M155 and K464 is insensitive to cAMP binding (Figure 1A), one might assume that the rotation induced by K464E is not solely caused by the loss of a hydrogen bond to the backbone of M155. In line with this assumption, patch-clamp experiments revealed only small effects on the channel activation for the K464A mutant compared to the K464E mutant, also suggesting that the gating behavior in K464E is not solely caused by the loss of a hydrogen bond to the backbone of M155, which would also occur in the case of K464A.

The negatively charged residues E243, D244, and E247 on the S2/S3-linker are further potential interaction partners of K464. In the apo wild type channel, only infrequent salt-bridge interactions were found between K464 and E243 (in < $0.5 \%$ of all conformations) or D244 (in $6.9 \% \pm 1.1 \%$ of all conformations; Figure 3A). A salt-bridge interaction with E247 occurs in $20.1 \% \pm 2.4 \%$ of all conformations (Figure 3A). Upon cAMP binding the interaction frequencies to D244 (in $2.7 \% \pm 0.7 \%$ of all conformations; $p<0.01$ ) and E247 (in $12.1 \% \pm$ $1.6 \%$ of all conformations; $p<0.01$ ) are significantly reduced (Figure 3A). Thus, it seems reasonable to assume that repulsive electrostatic forces, likely occurring in the K464E mutant, contribute to channel activation, which, in turn, mimics the reduced interaction frequencies 
bioRxiv preprint doi: https://doi.org/10.1101/2020.09.21.305797; this version posted September 21, 2020. The copyright holder for this preprint (which was not certified by peer review) is the author/funder. All rights reserved. No reuse allowed without permission.

observed upon cAMP binding. This assumption is supported by results from patch-clamp experiments, in which the activation voltage is shifted towards more positive values in the K464E mutant relative to the wild type channel (Figure 2E, H).

Taken together, the side-chain of K464 in $\mathrm{mHCN} 2$ is predicted to interact with the backbone of M155 of an opposite subunit. In the K464E channel, this interaction is lost and the substitution is suggested to introduce electrostatic repulsive forces between the subunits. As a structural consequence, a rotation of the CL-CNBD relative to the apo wild type channel pore is induced, which agrees in terms of direction and magnitude with structural changes induced by cAMP binding to mHCN2 or homologous HCN channels (6). These findings explain why apo K464E mutants show similar effects on channel activation and deactivation during patch-clamp experiments like wild type mHCN2 in the presence of saturating [cAMP].

A

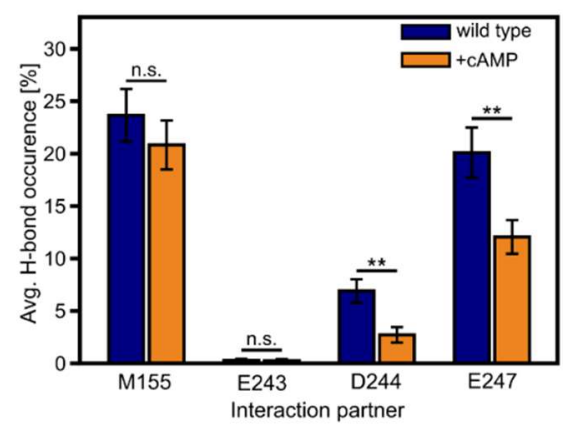

C

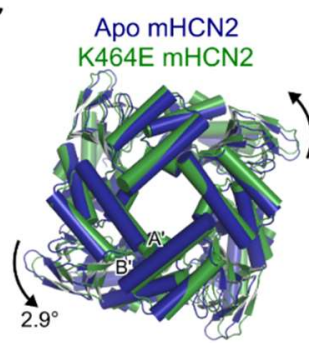

$B$

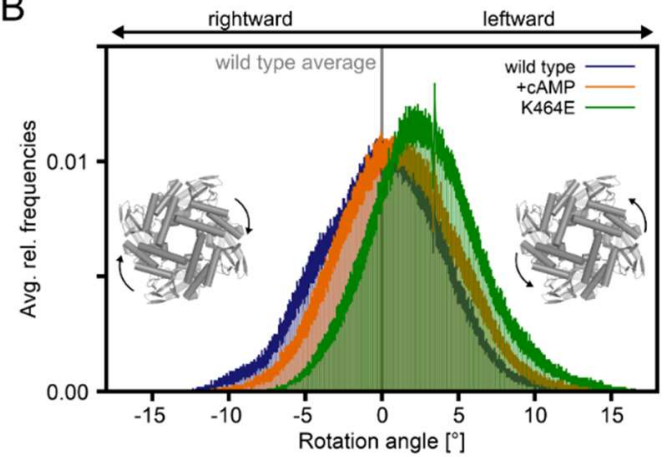

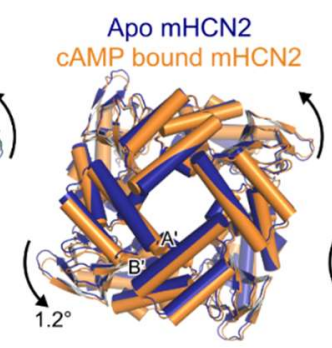

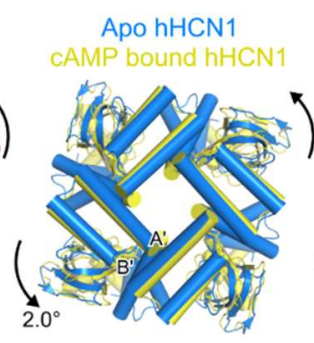

Apo hHCN4 cAMP bound hHCN4

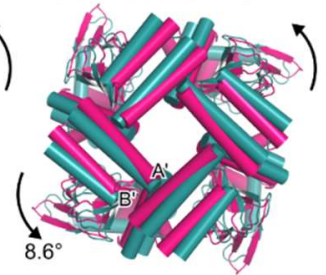

Figure 3: Analyses of MD simulations of the wild type and K464E mHCN2 channel.

A: Average occurrence frequency of hydrogen bond interactions between two opposite subunits involving K464. K464 resides on subunit $i$, and the interaction partners M150, E243, D244, and E247 reside on subunit $i+2$. As to M155, we only considered the backbone oxygen as H-bond acceptor; for E243, D244, and E247, we only considered the side-chain oxygen atoms, as we considered these interactions more favorable compared to backbone interaction. B: Histogram (bin size $0.1^{\circ}$ ) of the rotation angle relative to the channel pore normalized to the apo wild type average (the vertical line at $0^{\circ}$ ). Negative (positive) values indicate a rightward (leftward) rotation relative to the apo wild type structure, as indicated by the inset panels. C: Overlay of CL-CNBDs after superimposing the pore regions. From left to right, the panels show the overlay of the average apo wild type mHCN2 (dark blue) and the average K464E mHCN2 (green) or the average cAMP-bound wild type mHCN2 (orange) from MD simulations, the overlay of the apo (light blue, PDB 5U6O (6)) and cAMP-bound (yellow, PDB 5U6P (6)) cryo-EM structures of hHCN1, and the overlay of the apo (magenta, PDB 6GYN (17) and cAMP-bound (dark cyan, PDB 6GYO (21) cryo-EM structures of hHCN4. Helices are shown as cylinders. The arrows indicate the direction of rotation relative to the respective apo structures. The labels depict the angle of rotation relative to the respective apo structures. A'- and B'-labels denote the first two helices of the CL. In A and $\mathbf{B}$, the average values were calculated over all four subunits of the mHCN2 channels and 20 independent 
MD simulation runs ( $n=80$ independent replicas). The error bars denote the standard error of the mean (SEM). ( $p$ value by $t$-test; ** $p<0.01$; n.s. not significantly different).

\section{K464 acts via backbone but not via side-chain interaction with M155 of the opposite subunit}

To test the hypothesis that the backbone rather than the side chain of M155 is involved in hydrogen bond interactions with K464, we mutated M155 to alanine, arginine, or glutamate and showed the effects of these substitutions on steady-state activation. If the backbone-side chain interaction is the predominant interaction between M155 and K464, we expect only minor effects, if any, due to these mutations. All three constructs led to functional channels and robust currents. The results are summarized in Figure 4A and Figure S2.

M155A showed no difference in the steady-state activation relationship compared to mHCN2, neither in the absence of cAMP nor in the presence of saturating [cAMP] (Figure 4A, Figure S2), resulting in a similar $\Delta V_{1 / 2}$. In M155R, both relationships were slightly shifted to more hyperpolarized voltages, indicating a stabilization of the closed state. However, the cAMPinduced $\Delta V_{1 / 2}$ was in the range of wild type mHCN2. In M155E, both curves were shifted to more depolarized voltages, indicating a stabilization of the open state. Again, the effect on $\Delta V_{1 / 2}$ was minor. The slopes for all three mutants, representing the effective gating charges, were not different from those of the wild type channel (Figure S2).

Neither shortening the side-chain at position 155 (M155A) nor adding a positive charge (M155R) affected the gating behavior in a similar way as did K464E, which supports our idea that the side chain at position 155 is not interacting with the side chain of K464. Moreover, in M155E, analysis of steady-state activation revealed an open state-stabilization. If the introduced glutamate side chain interacted with the K464 side chain, we rather would expect a stabilization of the closed state. 


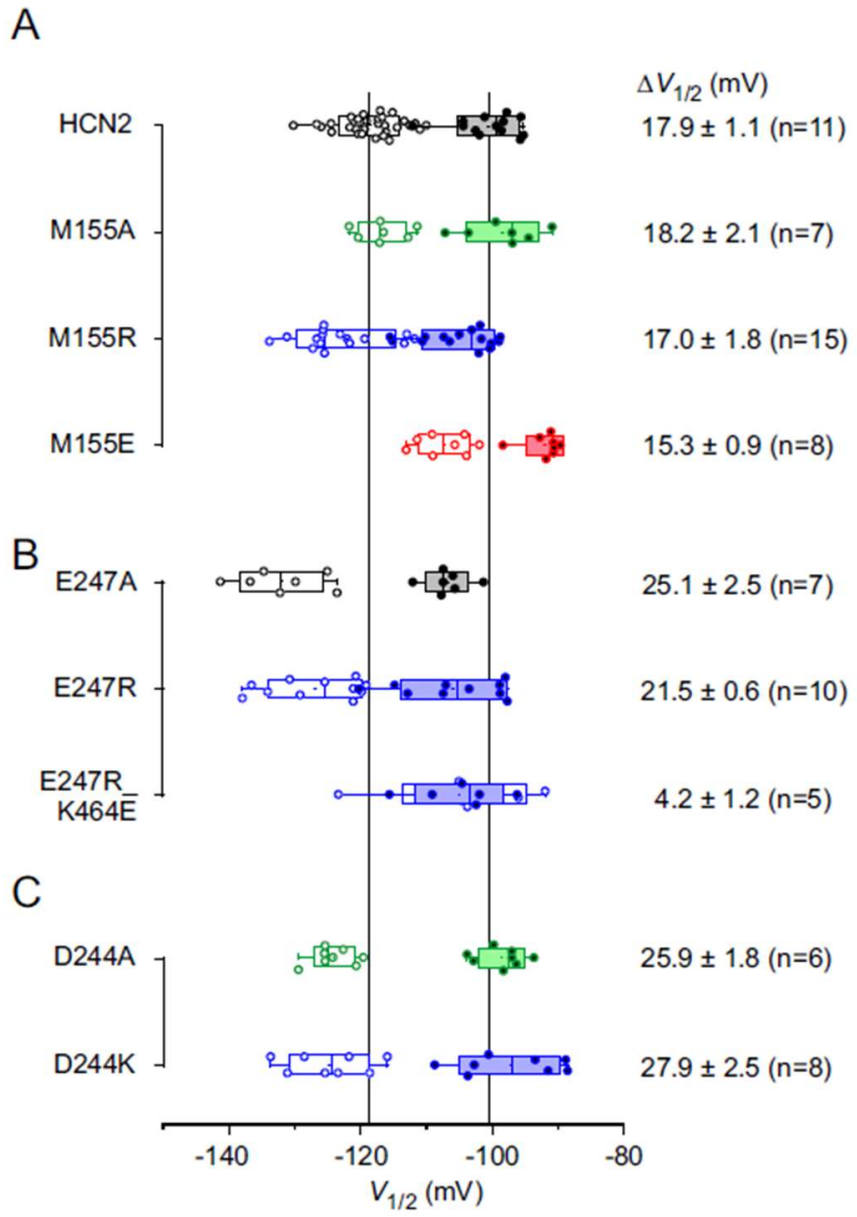

Figure 4: Voltage-dependent activation of potential interaction partners at zero and saturating [cAMP] (10 $\mu \mathrm{M})$.

Box plots indicating mean $V_{1 / 2}$ values and SD for M155 constructs (A), E247 constructs (B), and D244 constructs (C). Open and filled symbols represent individual recordings for zero and saturating cAMP, respectively. Dotted center lines represent median, box limits represent standard deviation, whiskers represent minimum and maximum values, cirles represent individual recordings, squares represent mean. Numbers give the cAMPinduced shift of $V_{1 / 2}, \Delta V_{1 / 2} \pm$ SEM. $\mathrm{n}$ indicates numbers of recordings, which were included in determining $V_{1 / 2}$. Only patches were included, in which both voltage families (with and without cAMP) could be recording successfully.

\section{Role of negatively charged residues in the S2-S3 loop for opposite subunit interactions}

As described above, E247 in the S2-S3 loop was identified as a potential interaction partner for K464 to form a salt bridge. Thus, we constructed the mutants E247A and E247R to study the role of this residue for channel gating. The data are summarized in Figure 4B. Surprisingly, cAMP-induced gating is not affected. However, there is an effect on voltage-induced gating: The steady-state activation relationships are shifted to more negative voltages for both mutants, indicating a stabilization of the closed state. This lends support to the above suggestion that the effects found for K464E and K464A are not solely due to breaking hydrogen bonds or saltbridges but also due to introducing electrostatic repulsive forces between the subunits. This result is further supported by the gating behavior of the mutant E247R_K464E. If a salt-bridge 
between K464 and E247 mediated the function of K464, such a bond should be rescued in E247R_K464E, leading to a wild type-like phenotype. However, in E247R_K464E, the cAMP dependence was still strongly reduced like in $\operatorname{K} 464 \mathrm{E}\left(V_{1 / 2}=4.2 \pm 1.2 \mathrm{mV}\right)$ (Figure 4B).

For D244, the probability of forming salt-bridge interactions with K464 is low (Figure 3A). We still tested the role of this residue for channel gating by constructing D244A and D244K. In both cases, $V_{1 / 2}$ values were shifted to more negative values in the absence of cAMP, while there were no changes for cAMP-saturated constructs (Figure 4C). Consequently, $\Delta V_{1 / 2}$ was rather increased than decreased. Thus, we can exclude D244 as a partner for forming an interaction with $\mathrm{K} 464$.

Together, these data suggest that the negatively charged residues in S2-S3 are not involved in cAMP-dependent gating.

\section{Pre-activated conformation of the CL-CNBD in K464A and K464E induces affinity change in CNBDs}

In $\mathrm{HCN}$ channels, a gating mechanism is proposed, in which for channel opening the CL-CNBD has to perform a leftward rotation relative to the channel pore to unwind the right-handed S6 helix bundle gate $(6,17)$. cAMP binding causes a leftward rotation of the CL-CNBD (Figure 3B, C). Without additional energy supplied by voltage, however, the cAMP-triggered rotation is not sufficient to open the channel; yet, it is supportive for the voltage-induced rotation (6). In this sense, cAMP causes the CL-CNBD to adopt a pre-activated or pre-disinhibited conformation.

In former studies employing patch-clamp fluorometry experiments, we and others showed that voltage-induced activation leads to an increase of cAMP binding affinity $(22,23)$. This increase of binding affinity preceded gate opening. Thus, it is not the actual gate opening that causes the affinity increase, but preceding conformational changes (22). However, the previous data could not be interpreted by underlying conformational changes.

The K464 mutants presented here provide a unique tool to probe if the leftward rotation of the CL-CNBD is the causative conformational change for the affinity increase. To this end, we measured the binding affinity of a fluorescently tagged cAMP derivative, 8-Cy3B-AHT-cAMP (f $f_{1}$ AMP), in K464E with confocal patch-clamp fluorometry (24). The results are summarized in Figure 5.

The representative confocal images in Figure 5A show patch pipettes carrying an excised macropatch expressing either wild type $\mathrm{mHCN} 2$ or K464E channels at a non-activating and an activating voltage of $-30 \mathrm{mV}$ and $-130 \mathrm{mV}$, respectively. The green fluorescence signal of the 
patch is caused by binding of $0.75 \mu \mathrm{M} \mathrm{f}_{1} \mathrm{cAMP}$ to the channels. The red signal in the background is caused by the reference dye Dy647 $(5 \mu \mathrm{M})$, used to subtract the background intensity of unbound 8-Cy3B-AHT-cAMP. Details of the subtraction procedure are described in the methods section and in ref. Biskup et al. (25).

To monitor the affinity increase in response to channel activation, we applied, in analogy to the patch-clamp only experiments, a voltage jump from -30 to $-130 \mathrm{mV}$, followed by a short test pulse of $-100 \mathrm{mV}$ and a deactivating pulse back to the holding potential of $-30 \mathrm{mV}$. For each patch the mean fluorescence intensity caused by a subsaturating $f_{1}$ cAMP concentration was quantified for the dome of the patch and normalized with respect to the individual maximum intensity caused by a saturating concentration of $2.5 \mu \mathrm{M}$, yielding $F / F_{\max }$. Representative intensity-time courses of $F / F_{\max }$, showing the ligand binding, and simultaneously measured current time courses, showing channel activation, are illustrated for K464E and mHCN2 in Figure 5B. There was no binding increase for K464E upon channel activation.

To quantify this in more detail, we plotted $F / F_{\max }$ against the $\mathrm{f}_{1} \mathrm{cAMP}$ concentration to yield concentration-binding relationships for -30 and $-130 \mathrm{mV}$ (Figure 5C). The Hill equation (equation 3) was fitted to the mean data yielding the concentration of half-maximum binding, $B C_{50}$, of $0.40 \mu \mathrm{M}$ for non-activated $\mathrm{mHCN} 2$ at $-30 \mathrm{mV}, 0.24 \mu \mathrm{M}$ for activated $\mathrm{mHCN} 2$ at -130 $\mathrm{mV}, 0.28 \mu \mathrm{M}$ for non-activated $\mathrm{K} 464$ at $-30 \mathrm{mV}$, and $0.26 \mu \mathrm{M}$ for activated $\mathrm{K} 464$ at $-130 \mathrm{mV}$. The Hill coefficients, $H$, were $1.5,1.6,1.8$, and 1.8 , respectively. As shown previously, in $\mathrm{mHCN} 2$, the affinity in hyperpolarized channels at $-130 \mathrm{mV}$ was higher than for non-activated channels at $-30 \mathrm{mV}(22,23)$. In K464E, there was no difference in cAMP affinity between activated and non-activated channels. Both values were similar to the affinity of activated mHCN2 wild type channels.

To explain, we determined changes in structural fluctuations within the CL-CNBD upon cAMP binding from our MD simulations and compared these changes with those induced by the K464E substitution. To this end, we computed the root mean square fluctuation (RMSF) of the side chains, a measure for atomic mobility, including all residues of the CL-CNBD. The sidechain mobility is expressed relative to the apo wild type channel ( $\triangle \mathrm{RMSF}$; eq.(4)). cAMP binding and K464E substitution lead to an overall rigidification of the CL-CNBD compared to the apo wild type channel (Figure 5D). Mapping $\triangle$ RMSF onto the structure of the CL-CNBD reveals that, in particular, the $\mathrm{CL}$ region and the $\beta$-roll of the CNBD, encompassing parts of the cAMP binding site, become less mobile in both cases. The magnitudes of $\triangle$ RMSF are in excellent agreement in both cases (Figure S3). As a unique feature for cAMP binding, the Cterminus of helix $\mathrm{C}$ in the CNBD is significantly less mobile, which may also explain why this 
region is not resolved in the hHCN1 in the absence of cAMP (6). The data thus provide evidence that cAMP-binding to the CNBD is structurally and functionally coupled to the CL region. Inversely, the effect of CL modulation due to the K464E substitution is structurally and functionally coupled to the CNBD. Note that these changes in structural fluctuations are overlaid by a CL-CNBD rotation induced similarly by either the K464E substitution or cAMP binding (see above). The cumulative changes in structural dynamics of the CL-CNBD in the case of K464E thus generate a CL-CNBD state to which cAMP binding is more favorable. From these data, we conclude that the leftward rotation of the CL-CNBD, caused either by the movement of the voltage sensors or by weakening interactions between the elbow region of the CL-CNBD and the HCN domain of the opposite subunit, is causative for the high-affinity state of the cAMP binding sites. 


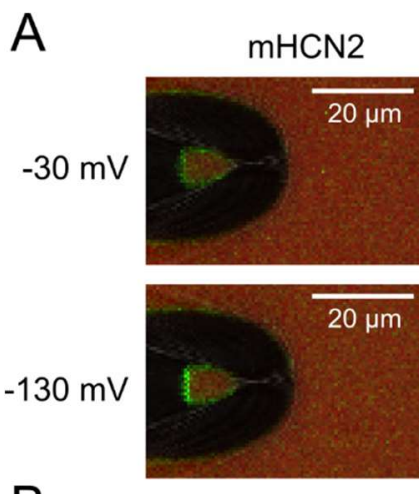

B
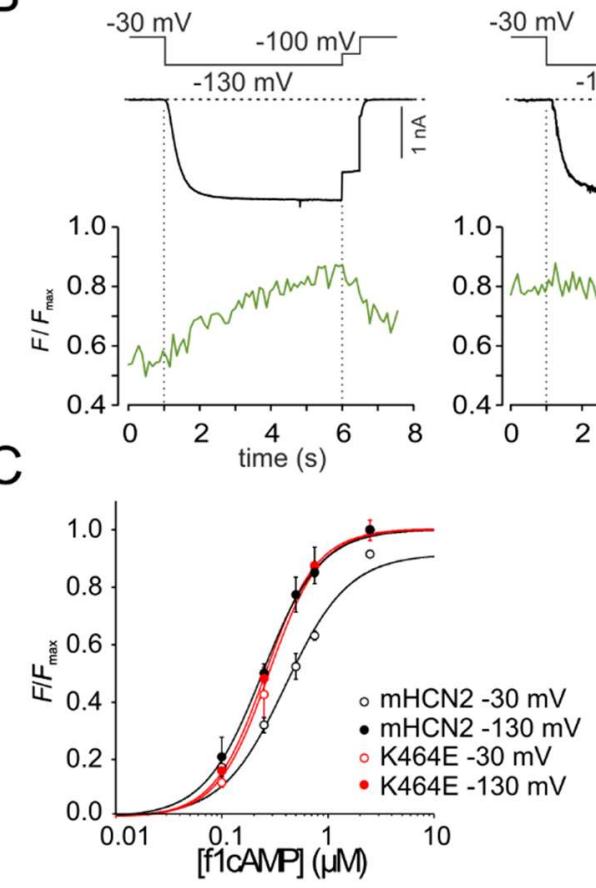

K464E
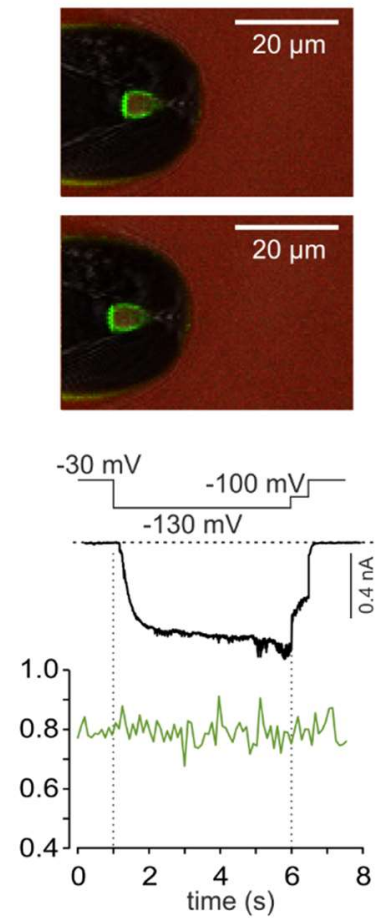

time (s)

D
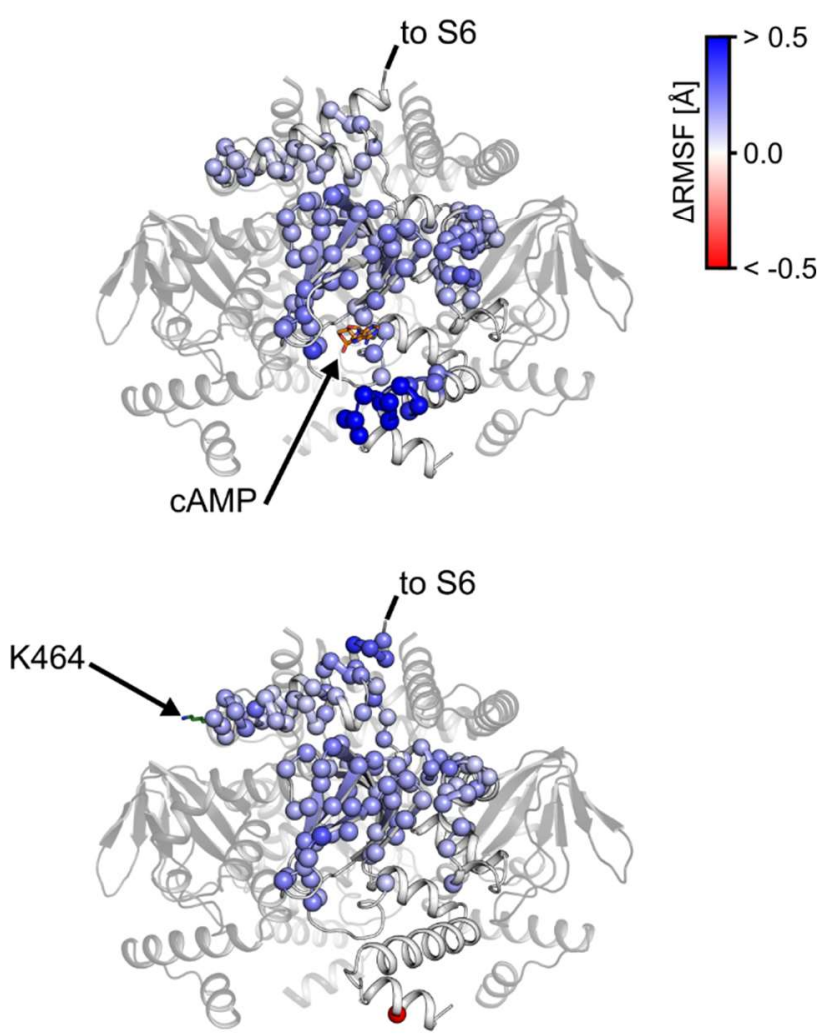
Figure 5: Activation-dependent affinities in K464 mutant and mHCN2 wild type channels.

A: Representative confocal images for $\mathrm{mHCN} 2$ and K464E. Upper panels show recordings at a non-activating voltage of $-30 \mathrm{mV}$, lower panels at activating voltage of $-130 \mathrm{mV}$. The tip of a patch pipette carrying a membrane patch expressing either mHCN2 or K464E is shown. The green signal is caused by $0.5 \mu \mathrm{M} 8$-Cy3B-AHT-cAMP binding to the channels, the red signal staining the background is caused by $5 \mu \mathrm{M}$ Dy647, a reference dye required for subtracting the background signal of unbound 8-Cy3B-AHT-cAMP. B: Time courses of simultaneously measured fluorescence increase (green) and current increase (black) following an activating voltage pulse from -30 to $-130 \mathrm{mV}$. C: Concentration-binding relationship for mHCN2 and K464E. The Hill function (equation 3 ) is approximated to the averaged data ( $\mathrm{n}=3$ to 6$)$ yielding the concentration of half-maximum binding, $B C_{50}$, and the Hill coefficient, $H$. D: Isolated CL-CNBD shown as cartoon. Residues that behave significantly different to apo wild type HCN2 are shown as spheres $\left(\mathrm{C}_{\alpha}\right.$ atoms only) and colored according to the residue-wise average $\triangle \mathrm{RMSF}$ (see color bar on the right; see also eq. (4); $n=80$ independent replicas). Residues colored in blue are significantly more mobile in the apo wild type channel ( $p<0.05 ; p$ value by $t$-test). Residues colored in red are significantly more mobile in the cAMP-bound wild type channel (top panel) or apo K464E channel (lower panel) $(p<0.05 ; p$ value by $t$-test). cAMP and K464 are shown as sticks.

\section{K464 is indirectly involved in gate modulation.}

It has been hypothesized that the rotation of the CL-CNBD could result in a displacement or unwrapping of the right-handed bundle of S6 helices, which harbors the gate-forming amino acids (6). As we recorded a rotation of the CL-CNBD induced by K464E, which is analogous to the rotation caused by cAMP binding $(6,17)$, we now analyzed our MD simulations towards changes of structural features of the gate region associated with the rotation. In the mHCN2 channel, the gate is formed by I432, T436, and Q440 (Figure 6A) which is identical to the architecture in hHCN4 (17) (I510, T514, and Q518) (Figure S4A). In hHCN1(6), by contrast, the isoleucine is substituted by valine, such that the gate is formed by V390, T394, and Q398 (Figure S4A). Because of the high structural similarity of isoleucine and valine, a comparison of the results across all three channels should still be appropriate.

To investigate whether the rotation of the CL-CNBD portion is associated with the gate opening, we measured the distances between two opposite gate residues of apo and cAMPbound wild type mHCN2, and K464E. One might assume that the CL-CNBD rotation induced by cAMP binding is associated with increased distances, although previous structural studies $(6,17)$ suggest only marginal changes in pore diameter (Figure S4B). Interestingly, also in the hyperpolarized conformation of hHCN1 the pore diameter remains almost unchanged, indicating a closed gate (26) (Figure S4B). In line with this observation, during MD simulations, the pore diameter neither changes significantly upon cAMP binding nor after K464E substitution (Figure 6B), suggesting a closed gate. Alternatively, CL-CNBD rotation, and thus unwrapping of the S6 helical bundle might increase the mobility of the gate-forming side chains I432, T436, and Q440 in mHCN2, which would lower the resistance that cations face when passing the gate. To prove this, we calculated the RMSF considering all nonhydrogen side-chain atoms of the gate-forming amino acids. Upon cAMP binding, the side 
chain of Q440 at the intracellular terminus of S6 is significantly more mobile compared to the apo wild type channel, whereas the opposite is seen for K464E (Figure 6C). Similar changes are observed for I432 and T436, with all but the differences between apo and cAMP-bound wild type channel being significant. Still, all differences are $<0.2 \AA$. We, thus, conclude that structural changes of the CL-CNBD due to cAMP binding or the K464E substitution observed in our MD simulations have no relevant impact on the gate conformation in HCN channels, similar to what is observed in comparative analyses of experimental HCN structures.

Previous structural and mutational studies indicated a complex interplay between helices S4, S5, and S6 of the transmembrane portion upon hyperpolarization and gate opening (26-29) (Figure S5A). Not surprisingly, in the absence of a hyperpolarizing voltage during MD simulations, the relative arrangement of helices S4 and S5 mimics that found under depolarized conditions in experimental structures (Figure S5B, C). As to the S5 and S6 helices, salt-bridges between these were found to be essential for stabilizing the closed channel, and alanine substitution of these residues favored channel opening $(27,29)$. In $\operatorname{mHCN} 2$, the salt-bridge between R339 and D443 is partially lost upon cAMP binding to mHCN2 $(58.78 \% \pm 3.99 \%$ in the apo wild type channel versus $46.19 \% \pm 4.33 \%$ in the cAMP-bound wild type channel; $p<$ $0.05)$, which is also the case in the K464E channel $(48.08 \% \pm 4.54 \% ; p=0.08$ relative to the apo wild type channel) (Figure 6D, E). The cAMP-bound wild type channel does not behave differently from K464E (Figure 6E). The reduced interaction frequency between D443 and R339 may thus provide one explanation of how cAMP binding or K464E destabilize the closed gate. 
A

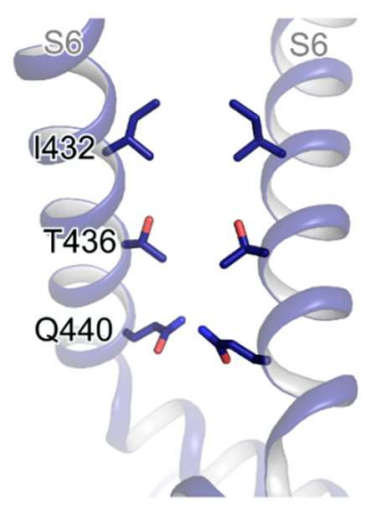

B

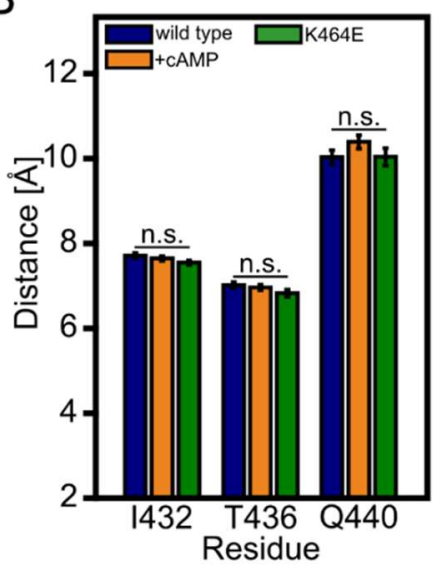

C

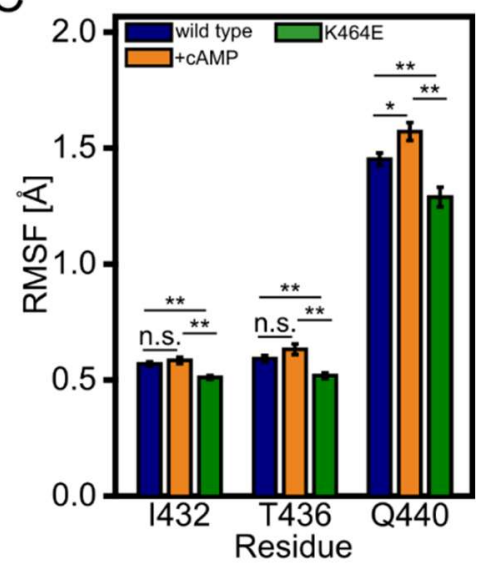

D

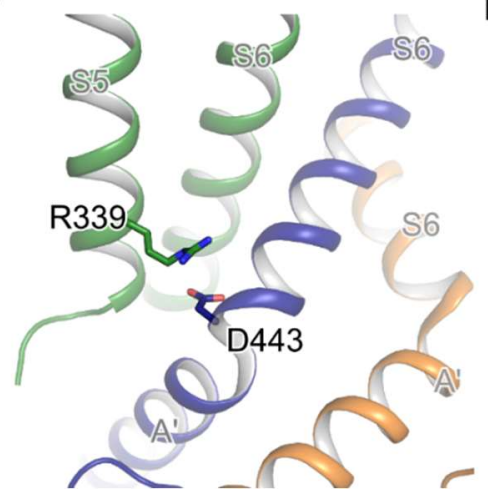

$\mathrm{E}$

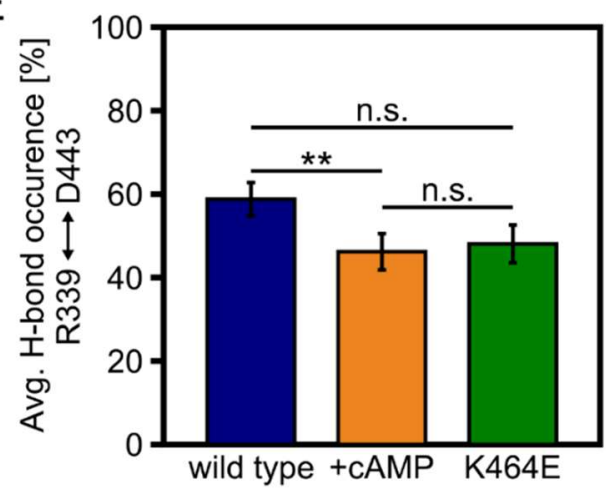

Figure 6: Conformational analyses of the S4/S5/S6 transmembrane portion of mHCN2.

A: The gate region of the mHCN2 channel pore with only two opposite subunits is shown. B: Distance measurements between the $\mathrm{C}_{\beta}$-atoms of two opposite amino acids that form the gate. $\mathbf{C}$ : Residue-wise root mean square fluctuations (RMSF) of gate-forming amino acids. All non-hydrogen side-chain atoms were considered. D: The close-up view depicts the suggested interaction between D443 on subunit $i$ (blue) and R339 on $i+1$ (green). E: Average occurrence frequency of salt-bridge interactions between residues depicted in panel D. In A and $\mathbf{D}$, relevant amino acids are shown as sticks. The bar plots in $\mathbf{B}, \mathbf{C}$, and $\mathbf{E}$ depict the average distance \pm standard error of the mean (SEM) or average occurrence frequency \pm SEM $(n=40$ independent replicas in $\mathbf{B}$ and $n=80$ independent replicas in $\mathbf{C}$ and $\mathbf{E})$. $\left({ }^{*} p<0.05\right.$; $* * p<0.01$; n.s. not significantly different; $p$ value by $t$ test). 


\section{DISCUSSION}

In this study, we addressed the question of whether a direct interaction of opposite subunits is essential for channel gating. To this end, we combined mutagenesis, patch-clamp, confocal patch-clamp fluorometry, and MD simulations. Previous studies proposed a gating mechanism, in which for channel opening the CL-CNBD performs a leftward rotation to unwind the righthanded S6 helix bundle gate (6). Such a leftward rotation is suggested to be induced by the movement of the voltage sensor upon a hyperpolarizing voltage jump. cAMP binding can cause a CL-CNBD leftward rotation, too. However, this cAMP-triggered rotation is not sufficient to open the channel but is supportive for voltage-induced rotation (6). In this sense, cAMP causes the CL-CNBD to adopt a "pre-activated" conformation.

Over the past years, a plethora of different residues has been identified that are involved in channel gating by mediating intra- and inter-subunit contacts. So far, only little is known about functionally relevant inter-subunit contacts between opposite subunits in HCN channels (15). Close inspection of the recently published hHCN1 channel structure (6) or a structural model of mHCN2 (Figure 1A) revealed the possibility that also opposite subunits might interact: The so-called elbow structure, a helix-turn-helix motif formed by the $\alpha$-helices A' and B' of the CL (14), is in close proximity to the HCN domain and the S2-S3 linker of the opposite subunit.

This elbow region is of particular interest as previous studies already suggested an essential role in channel activation via coupling the conformational changes in the CNBD to the channel pore $(10,30-33)$. It has been suggested that channel opening is related to a rotation of the CL around the central channel axis, due to the disruption of stabilizing interactions (34-36). Recently it was suggested that the N-terminal HCN domain plays a role in channel activation upon hyperpolarization and cyclic nucleotide binding $(15,37)$. However, the purpose of the $\mathrm{HCN} / \mathrm{CL}$ interaction on channel activation is not completely understood.

\section{Elbow tip keeps the channel closed}

At the very tip, we identified a lysine residue in $\mathrm{mHCN} 2, \mathrm{~K} 464$, which is a key player in controlling the CL-CNBD rotation. To study the elbow structure in detail, we either neutralized or reversed the positive charge by constructing the mutants K464A and K464E. In K464E, both steady-state and non-steady-state parameters suggested that in the absence of cAMP the closed state was destabilized, such that the empty K464E behaved like a cAMP-saturated wild type channel. In K464A, such a destabilization was visible to a lower degree, suggesting that interactions between negative charges foster closed-state destabilization. The CL-CNBD in K464E adopts a conformation similar to a cAMP-bound wild type channel, possibly by being 
pushed into a leftward rotation due to repulsive forces from the opposite subunit. To corroborate this hypothesis, we performed unbiased MD simulations of apo wild type mHCN2, wild type mHCN2 bound to four cAMP molecules, and the apo K464E variant of the mHCN2 channel. The simulations revealed that K464 forms a hydrogen bond with the backbone of M155 in the HCN domain, thereby bridging two opposite subunits. This hydrogen bond is significantly weakened upon K464 substitution in K464E. Furthermore, going from apo mHCN2 to mHCN2 bound to four cAMP molecules to the K464E variant, an increasing rotation angle of the CLCNBD in a leftward direction was found, which agrees in terms of direction and magnitude with structural changes induced by cAMP binding to homologous HCN channels (6).

The experimental structures of $\mathrm{hHCN} 1$ (6) and our MD simulations on mHCN2 revealed the backbone of M155 in the second $\alpha$-helix of the opposite HCN domain as an interaction partner. To exclude that the side-chain of M155 is involved in interactions with K464, we mutated the methionine to alanine, arginine, or glutamate and showed the effects of these substitutions on steady-state activation. The effects were only mild in all cases and, importantly, did not show the phenotype of K464E. We thus concluded that the side-chain of M155 is not involved in interactions with K464.

We next studied two negatively charged residues in the opposite S2-S3 linker, D244 and E247. Neither neutralization nor charge reversal at those positions resulted in phenotypes similar to K464A or K464E. We thus concluded that the function of K464 in mHCN2 wild type does not require the formation of a single interaction with either one of those residues. To further support this conclusion for the residue E247, which showed the highest likelihood of developing a saltbridge with K464, we confirmed with the mutant E247R_K464E that rescuing a possible saltbridge does not result in a wild type-like phenotype. This construct still behaved like the K464E mutant, possibly because repulsive forces from glutamate at position 464 do not only result from E247 but also from other negatively charged residues close by, compensating for the loss of E247. This finding may further suggest that D244 or E247 take over the role of an interaction partner of K464 if the respective other residue is substituted.

Notably, all four constructs showed a shift of $V_{1 / 2}$ to more negative values in the absence of cAMP, indicating a stabilization of the closed state. Because $V_{1 / 2}$ at saturating cAMP is less affected, for all constructs, the cAMP-induced $\Delta V_{1 / 2}$ is more pronounced than for mHCN2 wild type. Thus, the negatively charged residues in the S2-S3 linker seem to be involved in voltagedependent rather than in cAMP-dependent gating. 


\section{CL-CNBD rotation increases cAMP binding affinity}

All our results suggest that in the K464E channel the CL-CNBD is rotated in a leftward direction compared to an apo wild type channel. With this tool in our hands, we studied if this leftward rotation is crucial for inducing the activity-dependent increase of cAMP affinity (22, 23). To this end, we performed $\mathrm{cPCF}$ experiments in K464E employing a fluorescently tagged cAMP derivative, 8-AHT-Cy3B-cAMP (24). Interestingly, in K464E, there was no difference in cAMP affinity between activated and non-activated channels. Both cAMP affinities were similar to the cAMP affinity of activated mHCN2 wild type channels. We concluded that the leftward rotation of the CL-CNBD, caused by weakening interactions between the elbow region of the CL-CNBD and the HCN domain of the opposite subunit, is causative for the high-affinity state of the cAMP binding sites.

\section{cAMP-induced rotation does not affect the gate directly}

As we identified a rotation of the CL-CNBD portion induced by K464E analogous to the rotation caused by cAMP binding $(6,17)$, we used K464E as a tool to analyze the effect of this rotation on the inner S6 helix bundle gate. In our experiments, channel activation is favored upon cAMP binding or after $\mathrm{K}$ to $\mathrm{E}$ substitution. One might assume that this is related to a widening of the gate, which, in turn, facilitates cation passage of the gate more efficiently.

Indeed, by using the full-length hHCN1 structure, Gross and coworkers proposed a model in which, upon cAMP binding, the elbow moves to the shoulder of the adjacent subunit in an overall centrifugal motion away from the central axis of the channel pore, which might lead to a widening of the channel pore (33). However, the authors also stated that with their approach, they could not gauge whether the widening is sufficient to open the intracellular channel gate. Interestingly, our simulation data showed no direct modulation of the gate conformation, neither by K464E substitution nor upon cAMP binding. Thus, our simulation data mirrors the structural observations from high-resolution cryo-EM structures of the hHCN1 (6) and hHCN4 (17). Both channels were structurally resolved in the presence and absence of cAMP, and in neither of the channels did cAMP binding lead to a widening of the inner channel gate, underscoring the excellent agreement of simulation and experimental data. The latter result is in contrast to previous studies, reporting that cAMP-induced rotation of the CL disk leads to a consequent widening of the inner channel gate $(20,38)$. These studies have been performed on truncated channels lacking the transmembrane portion, however. In the most recent cAMP-bound hHCN1 
structure, where a hyperpolarized state was reached through chemical cross-linking, the gate also remains closed (26).

If there is no direct effect of cAMP-induced CL-CNBD rotation on the gate, what is the purpose of this rotation for HCN channel modulation? In HCN channels, the S6 helical bundle is in direct neighborhood to helices S5 and S4 $(6,17,26)$ (Figure S5). On the timescales of our MD simulations, we did not see a change in the spatial organization of these three helices, induced by neither cAMP nor K464E (Figure S5B). Comparative analyses of hHCN1 structures in apo and depolarized, cAMP-bound and depolarized, and cAMP-bound and hyperpolarized conformations confirm this observation $(6,26)$ (Figure S5A). By contrast, the conformations of helices S4 and S5 are highly sensitive to hyperpolarization $(6,26)$ (Figure S5A). However, in the absence of a hyperpolarizing voltage in our MD simulations, the relative arrangement of helices S4 and S5 mimics that found under depolarized conditions in experimental structures in all investigated states of mHCN2 (Figure S5B, C). A possible way for how cAMP binding- or K464E-induced CL-CNBD rotation can, at least indirectly, destabilize the closed gate is by reducing stabilizing interactions between R339 on S5 and D443 on S6 (Figure 6D, E). The inter-subunit salt-bridge between D443 and R339 locks the closed channel conformation (27, 29). CL-CNBD rotation, induced by either cAMP binding or K464E substitution, breaks the salt-bridge and, thus, removes or at least weakens the structural restraints of the channel gate, which, in turn, facilitates channel opening under hyperpolarization conditions. Recently, MD simulations of a truncated HCN channel, in which only the TM portion of the channel was considered, in the presence of a hyperpolarizing voltage recorded the S4 helix movement (28). Although not yet possible for full-length $\mathrm{HCN}$ channels, such simulations together with different rotation states of the CL-CNBD will pave the way to scrutinize the interplay of hyperpolarization-mediated activation and cyclic nucleotide-, or, as in the case of K464E, substitution-, mediated gating of HCN channels at the atomistic level.

In summary, opposite subunits in HCN channels are functionally linked to each other. Our data suggest that the interactions between opposite subunits maintain the autoinhibitory properties of HCN channels. Both cAMP binding and mutagenesis disrupt these interactions, driving the channel into a conformation, in which autoinhibition is weakened. We also identified residue $\mathrm{K} 464$, located on the $\mathrm{CL}$, as a pivotal player to maintain these interactions. 


\section{MARTERIALS \& METHODS}

\section{Xenopus laevis oocytes as heterologous expression system}

The surgical removal of oocytes was performed under anaesthesia $(0.3 \%$ tricaine methanesulfonate (MS-222) (Pharmaq Ltd. Fordingbridge, UK) from adult female South African claw frog Xenopus laevis. The oocytes were treated with collagenase A ( $3 \mathrm{mg} / \mathrm{ml}$; Roche, Grenzach-Wyhlen, Germany) for $105 \mathrm{~min}$ in $\mathrm{Ca}^{2+}$-free Barth's solution containing (in $\mathrm{mM}) 82.5 \mathrm{NaCl}, 2 \mathrm{KCl}, 1 \mathrm{MgCl}_{2}$, and 5 Hepes, $\mathrm{pH}$ 7.5. Oocytes of stages IV and $\mathrm{V}$ were manually dissected and injected with cRNA encoding either mHCN2 channels of Mus musculus or the mHCN2 mutants K464E, K464A, M155A, M155R, M155E, E247A, E247R, E247R_K464E, D244A, D244K, respectively. After injection with cRNA, the oocytes were incubated at $18^{\circ} \mathrm{C}$ for 2-6 days in Barth's solution containing (in $\mathrm{mM}$ ) $84 \mathrm{NaCl}, 1 \mathrm{KCl}, 2.4$ $\mathrm{NaHCO}_{3}, 0.82 \mathrm{MgSO}_{4}, 0.41 \mathrm{CaCl}_{2}, 0.33 \mathrm{Ca}\left(\mathrm{NO}_{3}\right)_{2}, 7.5$ TRIS, pH 7.4. Oocytes harvested in our own lab were complemented with ready-to-use oocytes purchased from Ecocyte Bioscience (Dortmund, Germany). The surgery procedures were carried out in accordance with the German Animal Welfare Act with the approval of the Thuringian State Office for Consumer Protection on 30.08.2013 and 09.05.2018.

\section{Molecular biology}

The mouse HCN2 (UniProt ID 088703 including two modifications, G8E and E55G without functional relevance) and all modified subunit variants were subcloned in front of the T7 promoter of pGEMHEnew. Point mutations K464E, K464A, M155A, M155R, M155E, E247A, E247R, E247R_K464E, D244A, D244K were introduced via the overlapping PCR-strategy followed by subcloning of the modified fragment using flanking restriction sites. Correctness of the sequences was confirmed by restriction analysis and sequencing (Microsynth SEQLAB, Göttingen, Germany). cRNAs were prepared using the mMESSAGE mMACHINE T7 Kit (Ambion Inc, Austin, USA).

\section{Electrophysiological experiments}

Macroscopic currents were recorded using the patch-clamp technique in the inside-out configuration. All measurements were started after a delay of 3.5 min to minimize run down phenomena. Patch pipettes were pulled from quartz tubings whose outer and inner diameter were 1.0 and $0.7 \mathrm{~mm}$ (VITROCOM, New Jersey, USA), respectively, using a laser puller (P2000, Sutter Instrument, Novato, USA). The pipette resistance was 1.2-2.1 MOhm. The bath solution contained (in mM) $100 \mathrm{KCl}, 10$ EGTA, and 10 Hepes, pH 7.2, and the pipette solution 
contained (in $\mathrm{mM}$ ) $120 \mathrm{KCl}, 10$ Hepes, and $1.0 \mathrm{CaCl}_{2}, \mathrm{pH} 7.2$. For parts of the experiments, a saturating concentration of $10 \mu \mathrm{M}$ cAMP (BIOLOG LSI GmbH \& Co KG, Bremen, Germany) was applied with the bath solution. An HEKA EPC 10 USB amplifier (Harvard Apparatus, Holliston, USA) was used for current recording. Pulsing and data recording were controlled by the Patchmaster software (Harvard Apparatus, Holliston, USA). The sampling rate was $5 \mathrm{kHz}$. The holding potential was generally $-30 \mathrm{mV}$. Each recording was performed in an individual membrane patch. Maximally two membrane patches were excised from one individual oocyte. For steady-state activation curves relative current values for each recording were fitted individually (see Quantification and statistical analysis).

\section{Confocal patch-clamp fluorometry}

The binding of the fluorescently tagged cAMP derivative 8-Cy3B-AHT-cAMP ( $f_{1}$ cAMP) (24)and the ionic current in macropatches were measured simultaneously by confocal patchclamp fluorometry (cPCF) as described previously $(22,25)$. The patch pipettes were pulled from borosilicate glass tubing with an outer and inner diameter of 2.0 and $1.0 \mathrm{~mm}$, respectively (Hilgenberg GmbH, Malsfeld, Germany). The pipette resistance was 0.7-1.2 M $\Omega$. The bath solution contained (in $\mathrm{mM}$ ) $100 \mathrm{KCl}, 10$ EGTA, and 10 Hepes, $\mathrm{pH} 7.2$, and the pipette solution contained (in $\mathrm{mM}$ ) $120 \mathrm{KCl}, 10 \mathrm{Hepes,}$ and $1.0 \mathrm{CaCl} 2, \mathrm{pH} 7.2$. Ionic currents were recorded using an Axopatch 200B amplifier (Axon Instruments, Foster City, USA). Current measurements were controlled and data were collected with the ISO3 software (MFK, Niedernhausen, Germany). The sampling rate was $2 \mathrm{kHz}$. Fluorescence imaging was performed with an LSM 710 confocal microscope (Zeiss, Jena, Germany) and was triggered by the ISO3 software (MFK, Niedernhausen, Germany). To subtract the fluorescence of the unbound $\mathrm{f}_{1} \mathrm{cAMP}$ from that of the bound $\mathrm{f}_{1} \mathrm{cAMP}$, a second dye, DY647 (Dyomics, Jena, Germany), was added to the bath solution at a concentration of $5 \mu \mathrm{M}$. $\mathrm{f}_{1} \mathrm{cAMP}$ and DY647 were excited at 543 $\mathrm{nm}$ and $633 \mathrm{~nm}$, respectively and detection bands of 546-635 nm and 637-759 nm were selected. Before subtraction, DY647 fluorescence intensity was scaled on $\mathrm{f}_{1} \mathrm{cAMP}$ fluorescence intensity in the bath and the pipette interior. The fluorescence intensity in the patch dome only was used to quantify the portion of bound ligands (25). A concentration of $2.5 \mu \mathrm{M} \mathrm{f}_{1} \mathrm{cAMP}$ was used for saturating all four binding sites and thus, for quantifying the maximum fluorescence, $F_{\max }$. For concentration-binding curves each membrane patch was exposed to the saturating concentration and, additionally, to one, two, three or four subsaturating concentrations. The resulting relative fluorescence intensities were averaged and the averaged values were fitted (see Quantification and statistical analysis). 


\section{Quantification and statistical analysis}

Steady-state activation curves were analyzed by fitting the Boltzmann equation to each individual recording using the OriginPro 9.0G software (Northampton, USA):

$$
I / I_{\max }=I / I_{\max , \operatorname{sat} V} /\left[1+\exp \left(z \delta F\left(V-V_{1 / 2}\right) / R T\right)\right]
$$

$I / I_{\max }$ is the relative current, $I / I_{\max , \text { satV }}$ is the relative current at a saturating voltage and the actual cAMP concentration, $V_{1 / 2}$ is the voltage of half-maximum activation and $z \delta$ the effective gating charge. $F, R$ and $T$ are the Faraday constant, the molar gas constant and the temperature in Kelvin, respectively.

The time courses of current activation and deactivation were fitted with a single exponential starting after an initial delay using the OriginPro 9.0G software (Northampton, USA).:

$$
I(t)=\mathrm{A} \exp [-t / \tau]
$$

$\mathrm{A}$ is the amplitude, $t$ the time, and $\tau$ the time constant for activation and deactivation, respectively.

Concentration-binding relationships were analyzed by approximating the Hill equation to averaged data using the OriginPro 9.0G software (Northampton, USA).

$$
F / F_{\max }=1 /\left[1+\left(B C_{50} /[\text { agonist }]\right)^{H}\right]
$$

$F$ is the actual fluorescent intensity at a given $\mathrm{f}_{1}$ cAMP concentration, $F_{\max }$ the maximal fluorescent intensity at a saturating concentration of $2.5 \mu \mathrm{M} \mathrm{f} \mathrm{f}_{1} \mathrm{cAMP}, B C_{50}$ the concentration of half-maximum binding, and $H$ the Hill coefficient of binding. Fluorescent intensities were generally obtained from the steady-state phase of a voltage pulse. Values for $B C_{50}$ and $H$ were yielded once for the averaged $F / F_{\max }$ data.

Experimental data are given as mean \pm standard error of mean (SEM). Statistical analysis was performed by an unpaired Student's t-test. A value of $\mathrm{p}<0.05$ was accepted as statistically significant.

\section{System setup for molecular dynamics simulations}

As currently no experimental 3D structure of the full-length mHCN2 is available, we carried out MD simulations using a homology model of mHCN2 (amino acid sequence taken from UNIPROT: O88703) based on the homologous and ligand-free hHCN1 structure (6) (PDB: $5 \mathrm{U} 6 \mathrm{O}$ ); for generating the homology model, Maestro ${ }^{\circledR}$ from the Schrödinger suite for molecular modeling (release 2018-3)(39) was used. The mHCN2 structure was built for the 
sequence from L136 to D650, and the sequence identity between mHCN2 and hHCN1 is $80 \%$. As to the CNBD of the ligand-free hHCN1 structure (6), the structures of helices C and D were not resolved completely, such that these two helices were prepared on the basis of the ligandbound hHCN1 (6) and afterwards connected to the rest of the mHCN2 structure. Thus, the final homology model of mHCN2 contains the HCN domain, the transmembrane helices, the CL, and the CNBD up until helix D.

The full-length mHCN2 was further prepared for simulations by using the Protein Preparation Wizard (40) distributed with Maestro ${ }^{\circledR}$ (release 2018-3)(39). First, we added ACE and NME groups to the channel's termini to avoid artificially charged termini. Protonation states were assigned according to the physiological $\mathrm{pH}$ of 7.4 and $\mathrm{pKa}$ values computed by PROPKA (41, 42). That way, all glutamate and aspartate residues are deprotonated and negatively charged, all lysine and arginine residues are protonated and positively charged. As to histidine residues, H474 was assigned to the HIP state (net charge +1 with hydrogen atoms bound at both imidazole nitrogen atoms), H178, H328, and H397 to the HIE state (net charge \pm 0 with a hydrogen atom bound at the $\varepsilon$-nitrogen atom), and the remaining histidine residues to the HID state (net charge \pm 0 with a hydrogen atom bound at the $\delta$-nitrogen atom). All hydrogen atoms were added according to the Amber ff14SB library (43). Finally, the prepared mHCN2 structure was assembled to a homotetrameric channel, again by using the $3 \mathrm{D}$ structure of the ligand-free hHCN1(6) as the template. The homotetrameric mHCN2 channel was inserted into a DOPC bilayer by using PACKMOL-Memgen (44). After adding $0.4 \mathrm{mM} \mathrm{KCl}$, the systems were solvated with SPC/E water (45), also by using PACKMOL-Memgen (44) (this structure is further referred to as the wild type mHCN2 channel). Initial coordinates for cAMP molecules were adapted from cAMP-bound structure of the hHCN1 channel. Therefore, we first aligned the CNBD backbone of the cAMP-bound hHCN1 structure to the ligand-free hHCN1 structure and translated the cAMP coordinates accordingly. As the differences between the two structures are small, no structural clashes between any cAMP atom and any channel atom was observed. To investigate also the influence of the K464E mutation on mHCN2 channel activation and deactivation, we also prepared a mHCN2 structure carrying the K464E mutation (this system is further referred to as the $\mathrm{K} 464 \mathrm{E}$ mHCN2 channel).

\section{Molecular dynamics simulations}

MD simulations were performed using Amber18 (46). The Amber ff14SB force field (43) was used for the mHCN2 channels, lipid17 for the DOPC lipids, parameters from Joung and Cheatham (47) for $\mathrm{K}^{+}$and $\mathrm{Cl}^{-}$, and SPC/E (45) for the water molecules. 
The detailed minimization, thermalization, and equilibration protocol is reported in ref. Kater et al. (48). In short, all structures were initially subjected to three rounds of energy minimization to resolve steric clashes. The system was then heated to $300 \mathrm{~K}$, and the pressure was adapted to 1 bar. During thermalization and pressure adaptation, we kept the protein atoms fixed by positional restraints of $1 \mathrm{kcal} \mathrm{mol}^{-1} \AA^{-2}$, which were gradually removed. Finally, an NPT simulation at $300 \mathrm{~K}$ and 1 bar of $50 \mathrm{~ns}$ length was performed with the unrestrained systems. Using the resulting structures as starting points, we performed 20 independent NPT production simulations at $300 \mathrm{~K}$ and 1 bar for $1 \mu$ s each. The initial velocities were randomly assigned during the first step of the NPT production simulation, such that each simulation can be considered as an independent replica. During production simulations, Newton's equations of motion were integrated in $4 \mathrm{fs}$ intervals, applying the hydrogen mass repartitioning approach (49) to all non-water molecules. Water molecules, by contrast, were handled by the SHAKE algorithm (50). Coordinates were stored into a trajectory file every 200 ps. The equilibration simulations were performed using the pmemd.MPI (51) module from Amber18 (46), while the production simulations were performed with the pmemd.CUDA module (52).

\section{Trajectory analysis}

All trajectories were analyzed with cpptraj (53) from AmberTools18. To investigate the interactions between two opposite subunits, we measured hydrogen bond interactions between K464 from one subunit to M155, E243, D244, and E247 from the other subunit. Hydrogen bond interactions were determined using a distance of $3.5 \AA$ between the two donor and acceptor atoms and an angle (donor atom, $\mathrm{H}$, acceptor atom) of $120^{\circ}$ as cutoff criteria (54).

To investigate the rotation of the CL-CNBD relative to the channel pore, but also relative to the starting structure, we measured the dihedral angle as indicated in Figure S6. Technically, the four reference points to define a dihedral angle were defined as follows: I) the center of mass (COM) of $\mathrm{C}_{\alpha}$-atoms of the four terminal residues (M460-H463) of the A'-helix of the CL, II) the $\mathrm{COM}$ of $\mathrm{C}_{\alpha}$-atoms of $\mathrm{T} 436$ on the $\mathrm{S} 6$ helix of each subunit, III) the $\mathrm{COM}$ of $\mathrm{C}_{\alpha}$-atoms of I432 on the S6 helix of each subunit, and IV) again the COM of $\mathrm{C}_{\alpha}$-atoms of the four terminal residues (M460-H463) of the A'-helix of the CL. The reference points I - III are static and were defined on the basis of the starting structure, while the reference point $\mathbf{I V}$ was determined over the course of the trajectory. That way, after superimposing the channel pore, a movement of IV will change the dihedral angle and can be recorded as a rotation of the CL-CNBD. Finally, we also calculated the average structures over the full ensemble of apo and cAMP-bound wild type mHCN2 and the K464E variant. 
To investigate changes in side-chain mobility, we calculated the residue wise root mean square fluctuation (RMSF), including all non-hydrogen side-chain atoms. To determine the changes relative to apo wild type $\mathrm{HCN} 2$, we calculated $\triangle \mathrm{RMSF}$ according to eq. (4)

$$
\left.\Delta \mathrm{RMSF}=\mathrm{RMSF}_{\text {apo, }} \text { wild type }-\mathrm{RMSF}_{\{\mathrm{cAMP}} \text {, wild type } \mid \text { apo } \mathrm{K} 464 \mathrm{E}\right\},
$$

where $\mathrm{RMSF}_{\mathrm{apo}}$, wild type is the mean residue wise RMSF of the apo wild type channel and $\mathrm{RMSF}_{\{\mathrm{cAMP}}$, wild type $\mid$ apo $\left.\mathrm{K} 464 \mathrm{E}\right\}$ is the mean residue wise RMSF of the cAMP-bound wild type

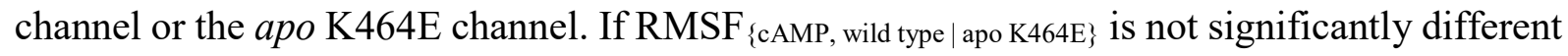
(in the case of $p>0.05 ; p$ value by $t$-test) to $\mathrm{RMSF}_{\text {apo, wild type, }} \Delta \mathrm{RMSF}$ was reset to zero, which allows focusing on the significant changes only.

To investigate gate modulation, we measured the distance between $\mathrm{C}_{\beta}$-atoms of two opposite amino acids that form the gate. In addition, we measured the RMSF of all non-hydrogen sidechain atoms of amino acids that form the gate. In mHCN2 the gate is formed by I432, T436, and Q440.

Hydrogen bond interactions, RMSF values, distances, and rotation angles are reported as mean value \pm SEM. As the mHCN2 channel is a homotetramer and as we performed 20 independent MD runs, the mean values and SEMs were calculated for $n=80$ independent replicas, if not stated differently. A two-sample $t$-test between mean values of apo and cAMP-bound wild type mHCN2 and the K464E variant was performed, and $p$-values $<0.05$ were considered significant.

\section{DATA AVAILABILITY}

The datasets generated during and/or analysed during the current study are available from the corresponding authors on reasonable request. 


\section{ACKNOWLEDGMENT}

We thank Sandra Bernhardt, Uta Enke, Andrea Kolchmeier, Claudia Ranke and Karin Schoknecht for excellent technical assistance. This work was supported by the DFG Research Unit 2518 DynIon of the Deutsche Forschungsgemeinschaft (projects P2 (KU 3092/2-1, BE 1250/19-1) and P7 (GO 1367/2-1)). We are grateful for computational support and infrastructure provided by the "Zentrum für Informations- und Medientechnologie" (ZIM) at the Heinrich Heine University Düsseldorf and the computing time provided by the John von Neumann Institute for Computing (NIC) to H.G. and B.F. on the supercomputer JUWELS at Jülich Supercomputing Centre (JSC) (user IDs: HKF7; HDD17).

\section{CONFLICT OF INTEREST}

The author(s) declare no competing interests.

\section{AUTHOR CONRIBUTIONS}

M.K. carried out electrophysiological and $\mathrm{CPCF}$ experiments and analyzed data. B.F. carried out the molecular simulation studies, analyzed data, prepared figures, and wrote the manuscript. S.Y. carried out electrophysiological and $\mathrm{cPCF}$ experiments. T.S. and C.S. engineered the mutants. M.L and A.S. synthesized fluorescent cylic nucleotides. R.S. designed experiments. K.B. designed experiments. H.G. designed molecular simulation studies, analyzed data, and wrote the manuscript. J.K. carried out electrophysiological and cPCF experiments and analyzed data, designed experiments, prepared figures, and wrote the manuscript. 


\section{REFERENCES}

1. Benarroch EE. HCN channels: function and clinical implications. Neurology. 2013;80(3):304-10.

2. Fenske S, Krause S, Biel M, Wahl-Schott C. The role of HCN channels in ventricular repolarization. Trends Cardiovasc Med. 2011;21(8):216-20.

3. Brown H, Difrancesco D. Voltage-clamp investigations of membrane currents underlying pace-maker activity in rabbit sino-atrial node. J Physiol. 1980;308:331-51.

4. DiFrancesco D, Tortora P. Direct activation of cardiac pacemaker channels by intracellular cyclic AMP. Nature. 1991;351(6322):145-7.

5. Mayer ML, Westbrook GL. A voltage-clamp analysis of inward (anomalous) rectification in mouse spinal sensory ganglion neurones. J Physiol. 1983;340:19-45.

6. Lee CH, MacKinnon R. Structures of the Human HCN1 Hyperpolarization-Activated Channel. Cell. 2017;168(1-2):111-20 e11.

7. Biel M, Wahl-Schott C, Michalakis S, Zong X. Hyperpolarization-activated cation channels: from genes to function. Physiol Rev. 2009;89(3):847-85.

8. Akimoto M, Zhang Z, Boulton S, Selvaratnam R, VanSchouwen B, Gloyd M, et al. A mechanism for the auto-inhibition of hyperpolarization-activated cyclic nucleotide-gated (HCN) channel opening and its relief by cAMP. J Biol Chem. 2014;289(32):22205-20.

9. VanSchouwen B, Akimoto M, Sayadi M, Fogolari F, Melacini G. Role of Dynamics in the Autoinhibition and Activation of the Hyperpolarization-activated Cyclic Nucleotidemodulated (HCN) Ion Channels. J Biol Chem. 2015;290(29):17642-54.

10. Wainger BJ, DeGennaro M, Santoro B, Siegelbaum SA, Tibbs GR. Molecular mechanism of cAMP modulation of HCN pacemaker channels. Nature. 2001;411(6839):80510 .

11. Kwan DC, Prole DL, Yellen G. Structural changes during HCN channel gating defined by high affinity metal bridges. J Gen Physiol. 2012;140(3):279-91.

12. Marni F, Wu S, Shah GM, Xu XP, Hackett AR, Xie C, et al. Normal-mode-analysisguided investigation of crucial intersubunit contacts in the cAMP-dependent gating in HCN channels. Biophys J. 2012;103(1):19-28.

13. Ulens C, Siegelbaum SA. Regulation of hyperpolarization-activated HCN channels by cAMP through a gating switch in binding domain symmetry. Neuron. 2003;40(5):959-70.

14. Zagotta WN, Olivier NB, Black KD, Young EC, Olson R, Gouaux E. Structural basis for modulation and agonist specificity of $\mathrm{HCN}$ pacemaker channels. Nature. 2003;425(6954):200-5.

15. Porro A, Saponaro A, Gasparri F, Bauer D, Gross C, Pisoni M, et al. The HCN domain couples voltage gating and cAMP response in hyperpolarization-activated cyclic nucleotidegated channels. Elife. 2019;8.

16. Sunkara MR, Schwabe T, Ehrlich G, Kusch J, Benndorf K. All four subunits of HCN2 channels contribute to the activation gating in an additive but intricate manner. J Gen Physiol. 2018;150(9):1261-71.

17. 6GYN [Internet]. The Protein Data Bank 2019.

18. Flynn GE, Zagotta WN. Insights into the molecular mechanism for hyperpolarizationdependent activation of HCN channels. Proc Natl Acad Sci U S A. 2018;115(34):E8086-E95.

19. James ZM, Zagotta WN. Structural insights into the mechanisms of CNBD channel function. J Gen Physiol. 2018;150(2):225-44.

20. Craven KB, Zagotta WN. Salt bridges and gating in the $\mathrm{COOH}$-terminal region of HCN2 and CNGA1 channels. J Gen Physiol. 2004;124(6):663-77.

21. $6 \mathrm{GYO}$ [Internet]. Protein Data Base. 2019. 
22. Kusch J, Biskup C, Thon S, Schulz E, Nache V, Zimmer T, et al. Interdependence of receptor activation and ligand binding in HCN2 pacemaker channels. Neuron. 2010;67(1):7585.

23. Wu S, Vysotskaya ZV, Xu X, Xie C, Liu Q, Zhou L. State-dependent cAMP binding to functioning HCN channels studied by patch-clamp fluorometry. Biophys J. 2011;100(5):122632.

24. Otte M, Schweinitz A, Lelle M, Thon S, Enke U, Yuksel S, et al. Novel Fluorescent Cyclic Nucleotide Derivatives to Study CNG and HCN Channel Function. Biophys J. 2019;116(12):2411-22.

25. Biskup C, Kusch J, Schulz E, Nache V, Schwede F, Lehmann F, et al. Relating ligand binding to activation gating in CNGA2 channels. Nature. 2007;446(7134):440-3.

26. Lee $\mathrm{CH}$, MacKinnon R. Voltage Sensor Movements during Hyperpolarization in the HCN Channel. Cell. 2019;179(7):1582-9 e7.

27. Decher N, Chen J, Sanguinetti MC. Voltage-dependent gating of hyperpolarizationactivated, cyclic nucleotide-gated pacemaker channels: molecular coupling between the S4-S5 and C-linkers. J Biol Chem. 2004;279(14):13859-65.

28. Kasimova MA, Tewari D, Cowgill JB, Ursuleaz WC, Lin JL, Delemotte L, et al. Helix breaking transition in the $\mathrm{S} 4$ of $\mathrm{HCN}$ channel is critical for hyperpolarization-dependent gating. Elife. 2019;8.

29. Nava C, Dalle C, Rastetter A, Striano P, de Kovel CG, Nabbout R, et al. De novo mutations in HCN1 cause early infantile epileptic encephalopathy. Nat Genet. 2014;46(6):6405 .

30. Weissgraeber S, Saponaro A, Thiel G, Hamacher K. A reduced mechanical model for cAMP-modulated gating in HCN channels. Sci Rep-Uk. 2017;7.

31. Prole DL, Yellen G. Reversal of HCN channel voltage dependence via bridging of the S4-S5 linker and post-S6. J Gen Physiol. 2006;128(3):273-82.

32. Flynn GE, Zagotta WN. Insights into the molecular mechanism for hyperpolarizationdependent activation of HCN channels. Proc Natl Acad Sci U S A. 2018;115(34):E8086-E95.

33. Gross C, Saponaro A, Santoro B, Moroni A, Thiel G, Hamacher K. Mechanical transduction of cytoplasmic-to-transmembrane-domain movements in a hyperpolarizationactivated cyclic nucleotide-gated cation channel. J Biol Chem. 2018;293(33):12908-18.

34. Johnson JP, Zagotta WN. Rotational movement during cyclic nucleotide-gated channel opening. Nature. 2001;412(6850):917-21.

35. Craven KB, Zagotta WN. Salt bridges and gating in the COOH-terminal region of HCN2 and CNGA1 channels. The Journal of General Physiology. 2004;124(6):663-77.

36. James ZM, Zagotta WN. Structural insights into the mechanisms of CNBD channel function. J Gen Physiol. 2018;150(2):225-44.

37. Cowgill J, Klenchin VA, Alvarez-Baron C, Tewari D, Blair A, Chanda B. Bipolar switching by $\mathrm{HCN}$ voltage sensor underlies hyperpolarization activation. Proc Natl Acad Sci U S A. 2019;116(2):670-8.

38. Craven KB, Olivier NB, Zagotta WN. C-terminal movement during gating in cyclic nucleotide-modulated channels. J Biol Chem. 2008;283(21):14728-38.

39. Schrödinger L. Schrödinger Release 2018-3: Schrödinger Suite 2018-3. Schrödinger, LLC, New York, NY. 2018.

40. Sastry GM, Adzhigirey M, Day T, Annabhimoju R, Sherman W. Protein and ligand preparation: parameters, protocols, and influence on virtual screening enrichments. J ComputAided Mol Des. 2013;27(3):221-34.

41. Sondergaard CR, Olsson MH, Rostkowski M, Jensen JH. Improved treatment of ligands and coupling effects in empirical calculation and rationalization of $\mathrm{pKa}$ values. J Chem Theory Comput. 2011;7(7):2284-95. 
42. Olsson MHM, Sondergaard CR, Rostkowski M, Jensen JH. PROPKA3: consistent treatment of internal and surface residues in empirical $\mathrm{pK}(\mathrm{a})$ predictions. J Chem Theory Comput. 2011;7(2):525-37.

43. Maier JA, Martinez C, Kasavajhala K, Wickstrom L, Hauser KE, Simmerling C. ff14SB: Improving the accuracy of protein side chain and backbone parameters from ff99SB. J Chem Theory Comput. 2015;11(8):3696-713.

44. Schott-Verdugo S, Gohlke H. PACKMOL-Memgen: A simple-to-use, generalized workflow for membrane-protein-lipid-bilayer system building. $\mathrm{J}$ Chem Inf Model. 2019;59(6):2522-8.

45. Berendsen HJC, Grigera JR, Straatsma TP. The missing term in effective pair potentials. J Phys Chem. 1987;91(24):6269-71.

46. Case DA, Ben-Shalom IY, Brozell SR, Cerutti DS, Cheatham I, T.E., Cruzeiro VWD, et al. AMBER 18. University of California, San Francisco. 2018.

47. Joung IS, Cheatham III TE. Determination of alkali and halide monovalent ion parameters for use in explicitly solvated biomolecular simulations. J Phys Chem B. 2008;112(30):9020-41.

48. Kater L, Frieg B, Berninghausen O, Gohlke H, Beckmann R, Kedrov A. Partially inserted nascent chain unzips the lateral gate of the Sec translocon. EMBO Rep. 2019:e48191. 49. Hopkins CW, Le Grand S, Walker RC, Roitberg AE. Long-time-step molecular dynamics through hydrogen mass repartitioning. J Chem Theory Comput. 2015;11(4):1864-74. 50. Ryckaert JP, Ciccotti G, Berendsen HJC. Numerical integration of cartesian equations of motion of a system with constraints molecular dynamics of n-alkanes. J Comput Phys. 1977;23(3):327-41.

51. Darden T, York DM, Pedersen LG. Particle Mesh Ewald: an N.log (N) method for Ewald sums in large systems. J Chem Phys. 1993;98(12):10089-92.

52. Salomon-Ferrer R, Götz AW, Poole D, Le Grand S, Walker RC. Routine microsecond molecular dynamics simulations with Amber on GPUs. 2. Explicit solvent particle mesh Ewald. J Chem Theory Comput. 2013;9(9):3878-88.

53. Roe DR, Cheatham III TE. PTRAJ and CPPTRAJ: software for processing and analysis of molecular dynamics trajectory data. J Chem Theory Comput. 2013;9(7):3084-95.

54. Desiraju GR, Steiner T. Weak hydrogen Bond: Oxford University Press New York; 2001. 